\title{
Drug resistance after cessation of efavirenz-based antiretroviral treatment started in pregnancy
}

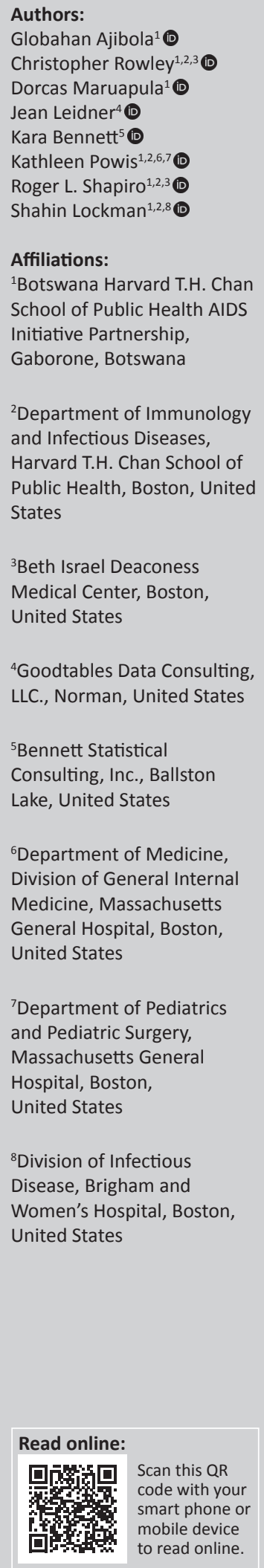

Background: To reduce risk of antiretroviral resistance when stopping efavirenz (EFV)-based antiretroviral treatment (ART), staggered discontinuation of antiretrovirals (an NRTI tail) is recommended. However, no data directly support this recommendation.

Objectives: We evaluated the prevalence of HIV drug resistance mutations in pregnant women living with HIV who stopped efavirenz (EFV)/emtricitabine (FTC)/tenofovir disoproxil fumarate (TDF) postpartum.

Method: In accordance with the prevailing Botswana HIV guidelines at the time, women with pre-treatment CD4 $>350$ cells $/ \mathrm{mm}^{3}$, initiated EFV/FTC/TDF in pregnancy and stopped ART at 6 weeks postpartum if formula feeding, or 6 weeks after weaning. A 7-day tail of FTC/TDF was recommended per Botswana guidelines. HIV-1 RNA and genotypic resistance testing (bulk sequencing) were performed on samples obtained 4-6 weeks after stopping EFV. Stanford HIV Drug Resistance Database was used to identify major mutations.

Results: From April 2014 to May 2015, 74 women who had stopped EFV/FTC/TDF enrolled, with median nadir CD4 of 571 cells $/ \mathrm{mm}^{3}$. The median time from cessation of EFV to sample draw for genotyping was 5 weeks (range: 3-13 weeks). Thirty-two (43\%) women received a 1-week tail of FTC/TDF after stopping EFV. HIV-1 RNA was available from delivery in 70 (95\%) women, 58 (83\%) of whom had undetectable delivery HIV-1 RNA ( $<40$ copies/mL). HIV-1 RNA was available for 71 women at the time of genotyping, 45 (63\%) of whom had HIV-1 RNA $<40$ copies / mL. Thirty-five (47\%) of 74 samples yielded a genotype result, and four $(11 \%)$ had a major drug resistance mutation: two with K103N and two with V106M. All four resistance mutations occurred among women who did not receive an FTC/TDF tail $(4 / 42,10 \%)$, whereas no mutations occurred among 18 genotyped women who had received a 1-week FTC/TDF tail ( $p=0.053)$.

Conclusions: Viral rebound was slow following cessation of EFV/FTC/TDF in the postpartum period. Use of an FTC/TDF tail after stopping EFV was associated with the lower prevalence of subsequent NNRTI drug resistance mutation.

Keywords: drug resistance; resistance mutations; HIV; antiretroviral treatment; Botswana.

\section{Introduction}

Cessation of antiretrovirals raises concerns about the potential for the development of drug-resistant viral strains, particularly for non-nucleoside reverse transcriptase inhibitors (NNRTIs). Non-nucleoside reverse transcriptase inhibitors, such as efavirenz (EFV), have long half-lives and a low barrier to the development of drug resistance. When used in combination with other classes of drugs such as nucleoside reverse transcriptase inhibitors (NRTIs), staggered discontinuation using a 4-7-day 'tail' of the NRTI backbone when stopping NNRTI-based antiretroviral treatment (ART) is advised. ${ }^{1}$ This is believed to mitigate the unintended period of monotherapy with NNRTIs that ensues because of the long half-life of most drugs in this class, thus reducing the risk of developing drug resistance. ${ }^{2,3}$ There are, however, very limited data to directly support this recommendation for a tenofovir disoproxil fumarate (TDF)/emtricitabine (FTC) 'tail': following cessation of EFV. Of note, pharmacokinetic variability (linked genetic factor-CYP2B6) in EFV metabolism between individuals has been observed, and EFV levels may

Corresponding author: Globahan Ajibola, gajibola@bhp.org.bw

Project research number: R01HD061265

Dates: Received: 19 Aug. 2019 | Accepted: 16 Sept. 2019 | Published: 27 Jan. 2020

How to cite this article: Ajibola G, Maruapula D, Rowley C, et al. Drug resistance after cessation of efavirenz-based antiretroviral treatment started in pregnancy. S Afr J HIV Med. 2020;21(1), a1023. https://doi.org/10.4102/sajhivmed.v21i1.1023 Copyright: (C 2020. The Authors. Licensee: AOSIS. This work is licensed under the Creative Commons Attribution License.

Note: For editorial commentary from Prof. Gary Maartens (University of Cape Town) on this article, please see: https://doi.org/10.4102/ sajhivmed.v21i1.1036. 
be higher in persons of African descent. ${ }^{4}$ These higher drug levels may also impact risk of drug resistance when stopping EFV-based ART.

Prior to 2015, both the World Health Organization (WHO) and the Botswana programme for the prevention of mother-to-child transmission (PMTCT) recommended that pregnant women living with HIV with a CD4 cell count > 350 cells $/ \mathrm{mm}^{3}$ without a WHO stage 3 or 4 illness take three-drug ART in pregnancy, but discontinue the ART postpartum or upon breastfeeding cessation (option B). Although both WHO and Botswana subsequently modified guidelines to recommend lifelong ART regardless of CD4 cell count or disease stage (option $\mathrm{B}+$ ), the prior guidance for pregnant women, as well as the inconsistent application of an NRTI tail at the time of EFV cessation, offers an opportunity to evaluate the development of resistance and the performance of a TDF/FTC tail among women stopping an $\mathrm{EFV} / \mathrm{TDF} / \mathrm{FTC}$ regimen. Because this ART regimen remains widely used throughout the world, the natural experiment afforded by prior PMTCT guidelines is applicable to our understanding of the risk of cessation of NNRTI-based regimens generally.

\section{Methods}

\section{Study population}

Between April 2014 and May 2015, we enrolled a subset of pregnant women living with HIV participating in a trial of infant cotrimoxazole prophylaxis in Botswana (the 'Mpepu' study) into this resistance sub-study. Mpepu was a double-blinded randomised controlled trial designed to assess the efficacy and safety of infant cotrimoxazole prophylaxis versus placebo used daily from as early as 14 days of life through 15 months among HIV-exposed uninfected infants in Botswana. ${ }^{5}$

Women were eligible for this sub-study of drug resistance following cessation of EFV/FTC/TDF if they were living with HIV, had pre-treatment CD4 $>350$ cells $/ \mathrm{mm}^{3}$ without evidence of WHO stage 3 or 4 disease, initiated EFV/FTC/ TDF in pregnancy and stopped ART postpartum according to Botswana guidelines at the time (at 4-6 weeks postpartum if formula feeding, or 6 weeks after weaning if breastfeeding). Per Botswana national HIV treatment guidelines, a 7-day tail of TDF/FTC after cessation of EFV was recommended. However, decisions regarding receipt and cessation of maternal ART occurred at government clinics (rather than study clinics), and the 7-day tail was inconsistently applied at the time of ART cessation.

\section{Data collection and laboratory testing}

Women eligible to participate in this sub-study were identified in the Mpepu study and scheduled for a clinic visit 4-6 weeks after ART cessation. Interested and eligible women consented to participate in the study and provided data (from self-report and medical records) on PMTCT regimen received; dates on which ART and individual antiretrovirals were started and stopped as well as provided blood specimens for drug resistance and HIV-1 RNA testing. HIV RNA results at the point of enrolment into the Mpepu study, and documented nadir CD4 counts, were retrieved and served as baseline values.

Prior to drug resistance testing, we first determined HIV-1 RNA viral load levels with the Abbott m2000sp/rt machine (limit of detection 40 copies/mL). Where HIV-1 RNA was detected, it was extracted using an automated validated technique. ${ }^{6}$ We then performed reverse transcription using in-house one-step RT-PCR technique. ${ }^{7}$ Polymerase chain reaction (PCR) products were then purified $^{8}$ and sequenced. ${ }^{9}$ Consensus sequences were generated, and the Stanford HIV Drug Resistance Database was used to identify all drug resistance mutations in the protease and reverse transcriptase coding regions.

\section{Statistical methods}

Statistical Package for Social Sciences (SPSS) version 25 was used to perform statistical analyses, which were generally descriptive in nature; a two-sided Fisher's exact test was used to evaluate for differences in the proportion of drug resistance by the approach to ART cessation, either with a 7-day TDF/FTC tail or with abrupt cessation of all three drugs. The small number of women with drug resistance mutations precluded more detailed analysis of predictors of drug resistance.

\section{Ethical considerations}

The Botswana Health Research Development Committee and the Office of Human Research Administration at Harvard T. H. Chan School of Public Health approved this drug resistance sub-study (HRDC 00732 and IRB13-2772), and women provided written informed consent for participation.

\section{Results}

\section{Baseline characteristics}

Ninety women enrolled, 74 of whom discontinued $\mathrm{EFV} / \mathrm{FTC} / \mathrm{TDF}$ postpartum and had samples collected for genetic resistance testing and are included in this analysis. Sixteen of 90 women not included in this analysis had CD4 $<350$ cell $/ \mathrm{mm}^{3}$ post-delivery and had to continue ART for their own health per Botswana national protocol at the time. The median time from cessation of EFV (whether or not a tail period occurred) to time sample was collected for resistance testing was 5 weeks (range: $3-13$ weeks). Median age at enrolment was 29 years (range: $20-45$ ) with median nadir CD4 count of 571 cells $/ \mathrm{mm}^{3}$ (range: 361-1236). Forty-seven (64\%) women had no previous exposure to antiretrovirals, $25(34 \%)$ reported previous exposure to antiretrovirals for PMTCT purposes in a prior pregnancy and $2(2 \%)$ were previously exposed to EFV/FTC/TDF but stopped prior to conception and then re-started EFV/FTC/TDF as three-drug prophylaxis for the index pregnancy. Thirty-two (43\%) stopped ART with the 
recommended 1-week tail of TDF/FTC after cessation of EFV and 42 (57\%) stopped all treatment at once. Of 70 women with viral load results at enrolment into the Mpepu study, 58 (83\%) had HIV-1 RNA < 40 copies/mL. At 4-6 weeks post-EFV/FTC/TDF cessation, 44 (62\%) of 71 women with viral load result had HIV-1 RNA $<40$ copies $/ \mathrm{mL}$. Thirty-three (47\%) women had HIV-1 RNA $<40$ copies/mL at delivery and at the time of sample draw for genotyping (post-ARV cessation).

\section{Mutations}

Thirty-five (47\%) of 74 samples were successfully sequenced, with $4(11 \%)$ of 35 having a major drug resistance mutation: 2 with K103N and 2 with V106M (Table 1). All four resistance mutations occurred among women who did not receive a TDF/FTC tail (4/42,10\%), whereas no mutations occurred among 18 genotyped women who had received a TDF/FTC tail ( $p=0.053)$. Eighteen $(58 \%)$ of the 31 women who had a genotype result and no drug resistance mutation took a TDF/FTC tail. Of 39 samples that could not be sequenced, 37 (95\%) had HIV-1 RNA $<40$ copies/mL in the sample being tested.

\section{Discussion}

A small proportion of women stopping EFV/FTC/TDF had a major NNRTI resistance mutation detected in plasma taken a little more than a month after stopping treatment. Among women receiving a 7-day tail of TDF/FTC after stopping EFV where genetic resistance testing was able to be performed, none had detectable mutations. Our findings thus support the recommended staggered discontinuation of antiretrovirals when an EFV/FTC/TDF regimen is being stopped. Our data support prior studies that evaluated the use of an NRTI tail when discontinuing EFV-based ART.

Our observation of prolonged viral suppression $<40$ copies / $\mathrm{mL}$ for a median of 5 weeks in $62 \%$ of women postART cessation was similar to findings from other studies which have demonstrated that it is not unusual for viral

TABLE 1: Characteristics of women with successful sequencing post-antiretroviral treatment cessation.

\begin{tabular}{|c|c|c|c|c|c|c|c|c|}
\hline Age (years) & $\begin{array}{l}\text { Prior use of ART } \\
\text { for own health }\end{array}$ & $\begin{array}{l}\text { Prior use of ART } \\
\text { for PMTCT }\end{array}$ & $\begin{array}{l}\text { Received } \\
\text { TDF/FTC tail }\end{array}$ & $\begin{array}{c}\text { Duration of ART } \\
\text { use (weeks) }\end{array}$ & $\begin{array}{l}\text { Nadir CD4 } \\
\text { (cell/UI) }\end{array}$ & $\begin{array}{c}\text { Baseline } \\
\text { VL (copies/mL) }\end{array}$ & $\begin{array}{l}\text { Post ART cessation } \\
\text { VL (copies/mL) }\end{array}$ & Mutation \\
\hline 27 & No & Yes & Yes & 31.6 & 518 & $<40$ & 42166 & E138A \\
\hline 27 & No & No & Yes & 38.4 & 570 & $<40$ & $<40$ & E138A \\
\hline 23 & No & No & Yes & 40.7 & 710 & $<40$ & 13120 & K103N \\
\hline 25 & No & No & Yes & 10.1 & 572 & $<40$ & 1719 & K103N \\
\hline 35 & No & No & No & 54.5 & 772 & $<40$ & 18259 & None \\
\hline 40 & No & Yes & Yes & 31.2 & 442 & $<40$ & 2658 & None \\
\hline 36 & No & Yes & No & 20.4 & 963 & $<40$ & $<40$ & None \\
\hline 25 & No & No & No & 20.2 & 611 & $<40$ & 857 & None \\
\hline 35 & No & No & No & 11.4 & 430 & $<40$ & - & None \\
\hline 25 & No & No & Yes & 15.8 & 575 & $<40$ & 33906 & None \\
\hline 24 & No & No & Yes & 25.2 & 496 & $<40$ & 5688 & None \\
\hline 26 & No & Yes & No & 25.6 & 460 & $<40$ & 2342 & None \\
\hline 27 & No & Yes & Yes & 14.0 & 908 & $<40$ & 1805 & None \\
\hline 29 & No & No & Yes & 23.1 & 436 & 58 & 379 & None \\
\hline 42 & No & No & Yes & 27.8 & 533 & $<40$ & $<40$ & None \\
\hline 40 & No & No & No & 13.5 & 361 & 52 & 250 & None \\
\hline 33 & Yes & No & Yes & 10.8 & 796 & $<40$ & $<40$ & None \\
\hline 30 & No & No & No & 21.1 & 616 & - & 9764 & None \\
\hline 26 & No & No & No & 24.9 & 1037 & $<40$ & 554 & None \\
\hline 27 & No & No & Yes & 16.1 & 601 & $<40$ & 3648 & None \\
\hline 40 & No & No & Yes & 24.6 & 363 & $<40$ & 33432 & None \\
\hline 27 & No & No & No & 81.6 & 673 & $<40$ & 196735 & None \\
\hline 24 & No & No & Yes & 39.2 & 800 & $<40$ & $<40$ & None \\
\hline 31 & Yes & No & No & 36.7 & 1236 & $<40$ & - & None \\
\hline 31 & No & Yes & Yes & 11.0 & 589 & 125 & $<40$ & None \\
\hline 29 & No & No & No & 18.5 & 715 & $<40$ & 2261 & None \\
\hline 26 & No & Yes & No & 96.2 & 612 & $<40$ & $<40$ & None \\
\hline 41 & No & No & No & 82.1 & 840 & - & 2229 & None \\
\hline 35 & No & Yes & No & 45.8 & 960 & $<40$ & 4536 & None \\
\hline 40 & No & Yes & No & 26.8 & 679 & $<40$ & 972 & None \\
\hline 28 & No & No & No & 21.9 & 436 & $<40$ & 40709 & None \\
\hline 26 & No & No & No & 23.2 & 397 & $<40$ & 309 & None \\
\hline 24 & No & No & No & 26.5 & 468 & 52 & $<40$ & None \\
\hline 20 & No & No & Yes & 22.4 & 384 & 45 & $68 \dagger$ & V106M \\
\hline 33 & No & Yes: & Yes & 23.8 & 468 & $<40$ & 145392 & V106M \\
\hline
\end{tabular}

ART, antiretroviral treatment; FTC, emtricitabine; TDF, tenofovir disoproxil fumarate; VL, viral load.

$\dagger$, Genotypes were performed independent of the knowledge of the participants' viral load; therefore, verification of the viral load on individual plasma sample was not performed.

$\$$, Previous exposure to zidovudine for prevention of mother-to-child transmission (PMTCT). 
suppression to persist for weeks after stopping ART. ${ }^{10}$ We believe this could be because of low pre-ART viral loads in our population (because of women with less advanced disease starting ART in pregnancy).

Our study had several limitations. Firstly, our results are based on a small number of observations making generalisability challenging. Secondly, we did not report on minor drug resistance variants and, finally, more than half $(63 \%)$ of women tested at a median time of 5 weeks from EFV cessation were still virally suppressed, which may suggest that we might have tested for resistance strain development early. Thirdly, we could not determine why the 7-day tail was inconsistently applied at the time of ART cessation and could therefore have missed evaluating possible confounders related to this factor.

\section{Conclusion}

Although guidelines no longer recommend discontinuation of ART after pregnancy, our study has general applicability for those requiring permanent or temporary treatment discontinuation when receiving an EFV-based regimen, informing the risk of mutation emergence in the setting of abrupt three-drug discontinuation versus discontinuing with a TDF/FTC tail. Furthermore, despite the shift to the use of newer drugs such as dolutegravir for which there is less concern about the emergence of resistance than with NNRTIs, our data are relevant to millions of individuals still on EFV-based ART (and are of particular clinical importance in regions that have access to only a limited number of antiretroviral drugs and that are also generally unable to routinely assess for drug resistance mutations before initiating a new ART regimen).

Based upon our results, we conclude that an TDF/FTC tail may help prevent selection of major NNRTI drug resistance mutations that can occur with the abrupt discontinuation of an EFV/FTC/TDF ART regimen.

\section{Acknowledgements}

Many thanks to the management and staff of the Botswana Harvard AIDS institute partnership for their support and to all the participants enrolled in the study.

\section{Competing interests}

The authors declare that no competing interests exist.

\section{Authors' contributions}

S.L. was the project leader, and G.A. and S.L. were responsible for experimental and project design. G.A. and C.R. performed most of the experiments. R.L.S. and K.P. made conceptual contributions, while D.M. prepared the samples. Calculations were performed by G.A., J.L. and K.B. G.A, C.R. and S.L. co-wrote the article.

\section{Funding information}

This project was funded by the National Institute of Child Health and Human Development, and the National Institute of Allergy and Infectious Diseases, NIH grant: R01HD061265.

\section{Data availability statement}

Data and associated documentation from this study will be made available for external use only under a data-sharing agreement that provides for (1) a commitment to using the data only for research purposes and not to identify any individual participant; (2) a commitment to securing the data using appropriate computer technology; and (3) a commitment to destroying or returning the data after analyses are completed.

\section{Disclaimer}

The views and opinions expressed in this article are those of the authors and do not necessarily reflect the official policy or position of any affiliated agency of the authors.

\section{References}

1. Lamorde M, Schapiro JM, Burger D, Back DJ. Antiretroviral drugs for prevention of mother-to-child transmission: Pharmacologic considerations for a public health approach. AIDS. 2014;28(17):2551-2563 https://doi.org/10.1097/ QAD.0000000000000439

2. McIntyre JA, Hopley M, Moodley D, et al. Efficacy of short-course AZT plus 3TC to reduce nevirapine resistance in the prevention of mother-to-child HIV transmission: A randomized clinical trial. PLoS Med. 2009;6(10):e1000172. https://doi.org/10.1371/journal.pmed.1000172

3. Chi $B H$, Sinkala $M$, Mbewe $F$, et al. Single-dose tenofovir and emtricitabine for reduction of viral resistance to non-nucleoside reverse transcriptase inhibitor drugs in women given intrapartum nevirapine for perinatal HIV prevention: An open-label randomised trial. Lancet. 2007;370(9600):1698-1705. https://doi. org/10.1016/S0140-6736(07)61605-5

4. Sinxadi PZ, Leger PD, Mcllleron HM, et al. Pharmacogenetics of plasma efavirenz exposure in HIV infected adults and children in South Africa. Br J Clin Pharmacol. 2015;80(1):146-156. https://doi.org/10.1111/bcp.12590

5. Lockman S, Hughes M, Powis K, Ajibola G, Bennett K, Moyo S. Effect of co-trimoxazole on mortality in HIV-exposed but uninfected children in Botswana (the Mpepu Study): A double-blind, randomized, placebo-controlled trial. Lancet Glob Health. 2017;5(5):e491-e500. https://doi.org/10.1016/\$2214-109X(17)30143-2

6. EZ1 Virus Mini kit v2.0, Virus Handbook. QIAGEN sample and assay technologies, USA [homepage on the Internet]. 2010 [cited 2019 Jul 04]. Available from: https://www.qiagen.com/cn/resources/download.aspx?id=ca13c92a-4b1b4ced-9796-b0414a166803\&lang=en

7. Rowley CF, MacLeod IJ, Maruapula D, et al. Sharp increase in rates of HIV transmitted drug resistance at antenatal clinics in Botswana demonstrates the need for routine surveillance. J Antimicrob Chemother. 2016;71(5):1361-1366. https://doi.org/10.1093/jac/dkv500

8. BigDye Terminator v3.1 Cycle Sequencing Kit User Guide. Thermo-Fisher scientific [homepage on the Internet]. 2016 [cited 2019 Jul 04]. Available from: http://tools.thermofisher.com/content/sfs/manuals/cms_081527.pdf

9. Applied Biosystems 3130/3130xl Genetic Analyzers User Guide. Life technologies [homepage on the Internet]. 2012 [cited 2019 Jul 04]. Available from: http://tools. thermofisher.com/content/sfs/manuals/4477796.pdf

10. Graham TC, Aga E, Bosch RJ, et al. Brief report: Relationship among viral load outcomes in HIV treatment interruption trials. J Acquir Immune Defic Syndr. 1999;72(3):310-313. https://doi.org/10.1097/QAl.0000000000000964 